\title{
Ocular findings in the acquired immunodeficiency syndrome
}

\author{
A. GAL, A. POLlaCK, And M. Oliver
}

From the Department of Ophthalmology, Kaplan Hospital, Rehovot, Israel, affiliated to the Hebrew University and Hadassah Medical School, Jerusalem, Israel

SUMmarY A patient with acquired immunodeficiency syndrome is described. Both retinae were severely affected. Vision was reduced to light perception with projection incerta in the right eye and finger counting at $1.5 \mathrm{~m}$ in the left eye. Eye involvement is common in this syndrome, appearing at an early stage of the disease. The fundal appearance could thus be of help in diagnosis.

A syndrome of acquired cellular immunodeficiency in previously healthy homosexual males has recently been described. ${ }^{1-3}$ These patients suffer from systemic opportunistic infections such as Pneumocystis carinii pneumonia, candidiasis, and different viral infections, of which cytomegalovirus (CMV) has been suspected of inducing the immunodeficiency. Kaposi's sarcoma has also been reported to occur in association with this syndrome. ${ }^{45}$ Ocular lesions are frequently found, affecting mainly the posterior segment of the eye. . $^{-9}$

We report here on a patient suffering from acquired immunodeficiency syndrome who had extensive involvement of vitreous and retina and ultimately became blind. The clinical findings and especially the fundal appearance are described.

\section{Case report}

A 24-year-old homosexual black male was admitted to a medical ward of Kaplan Hospital in October 1982. The previous history was based on the report submitted by his physician. He had been in good health until February 1982, when he developed fever, night sweating, weight loss, and dyspnoea. He was admitted to hospital elsewhere and $P$. carinii pneumonia was diagnosed. The pneumonia responded to treatment with trimethoprim, sulphamethotazole, and pentamidine. Lymphocytes, urine culture, and bronchial brushings were found to be positive for CMV. Anaemia and lymphopenia were evident. Immunological examination demonstrated cutaneous anergy and decreased in-vitro T-cell pro-

Correspondence to A. Gal, MD, Kaplan Hospital, 76-1(X) Rchovot, Isracl. liferation responses. In July 1982 the patient developed visual loss in the right eye. CMV retinitis was diagnosed. He was treated with a 2-week course of interferon intramuscularly (alpha- 2 recombinant) without benefit, and thereafter with one cycle of intravenous arabinoside-A. However, the vision did not improve. In August 1982 oral and oesophopharyngeal candidiasis developed, but it improved following administration of amphotericin-B. In September 1982 he developed progressive pulmonary insufficiency with right pleural effusion and a left solitary pulmonary nodule. Open lung biopsy was performed and the nodule resected. Histological examination revealed a pseudolymphoma, presumably occurring as a result of the histologically confirmed presence of CMV pneumonia. At that time the patient began to experience progressive visual loss in the left eye. He was transferred to our hospital for a therapeutic trial with thymic hormone factors in an attempt to improve his immunological status.

On admission in October 1982 the patient was debilitated and emaciated. Clinical examination revealed the presence of oral thrush and lymphadenopathy. The heart, lungs, and abdomen were normal. Vision was counting fingers from $1.5 \mathrm{~m}$ with the right eye and light perception projection incerta with the left. Goldmann's applanation tonometry revealed an intraocular pressure of $6 \mathrm{mmHg}$ in both eyes. Slit-lamp examination showed that the corneae, irides, and lenses were normal. In the right eye numerous cells were seen in the anterior vitreous. The fundus could not be clearly seen because of a heavy vitreous haze, particularly on the nasal side, where the retinal features could not be identified (Fig. 1). There was temporal pallor of the optic nerve. 


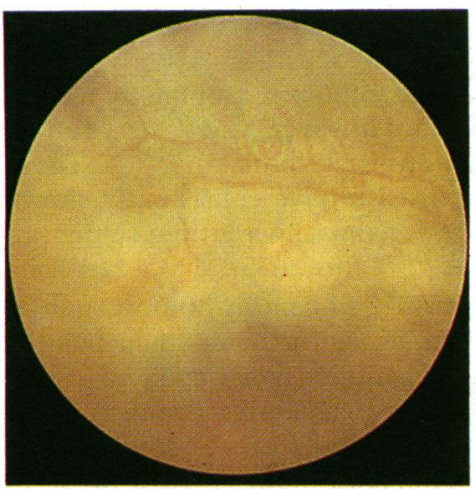

Fig. 1 Right eye. The vitreous is hazy. Extensive retinal infiltration is seen along vessels with perivascular sheathing in the area of the superior arcade.

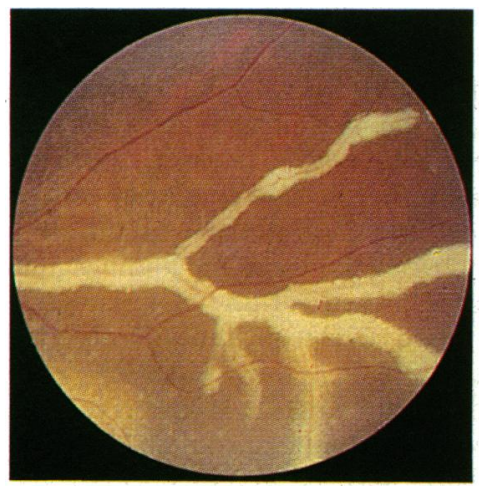

Fig. 4 Left eye. Perivascular sheathing of a superior temporal vessel with small cotton-woolspots.

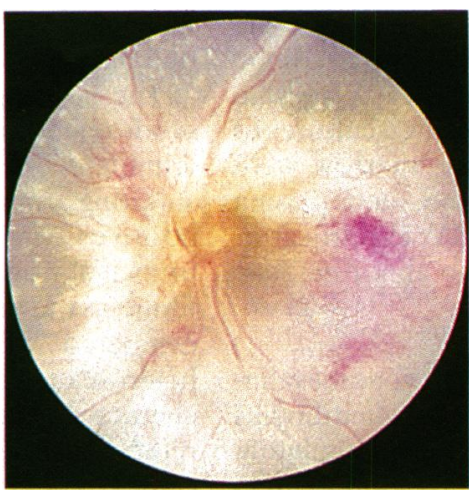

Fig. 2 Left eye. Extensive peripapillary retinal damage gives the appearance of 'ketchup on cottage cheese. 'There is narrowing of vessels and perivascular sheathing. Cotton-wool spots are present in the adjacent retina.

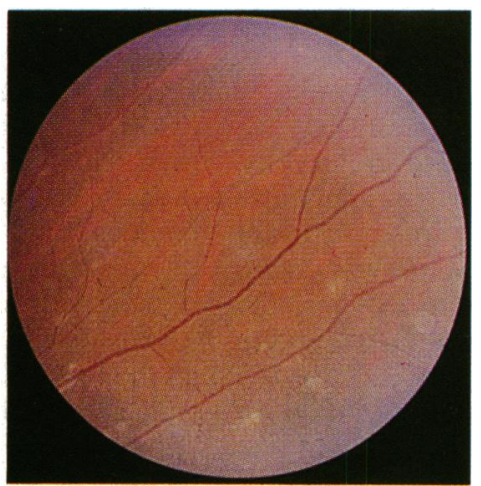

Fig. 5 Left eye. Peripheral retina with a few simall cotton-wool spots in an almost normal retina.

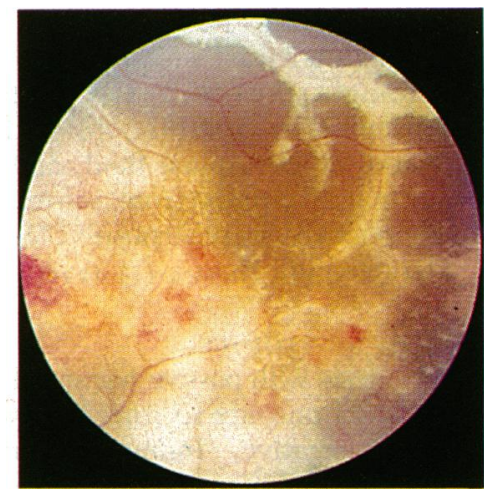

Fig. 3 Left eye. Retinal lesions are similar to those seen in Fig. 2. The severity of the affected retina diminishes towards the midperiphery. There is prominen perivasculitis.
Severe narrowing and focal sheathing of the lower major retinal vessels were observed. The upper branches appeared normal in the vicinity of the optic nerve but became sheathed and surrounded with infiltrates towards the periphery. These perivascular lesions had a yellowish, glittering appearance (Fig. 1). Wide confluent areas of white, thickened retina extended from the optic nerve along the upper vascular arcade towards the midperiphery. There was less involvement of the lower temporal half of the retina, including the lower part of the macula. The affected retina had a whitish appearance, and all the retinal layers appeared to be involved.

In the left eye the vitreous was clear. Examination of the fundus disclosed a severely oedematous optic nerve. The retinal lesions had the typical appearance of 'ketchup on cottage cheese,' with irregular borders and round satellite lesions in the adjacent retinal tissue. A few small superficial haemorrhages were observed (Fig. 2). The areas of involved retina extended from the optic disc, including the macular area, towards the midperiphery. The lower retina was more severely affected, and in some parts the lesions prevented visualisation of the retinal vessels (Figs. 2, 3). However, sheathing could be detected around both arterioles and venules, which were significantly narrowed (Fig. 2). The thickening and opacity of the retina diminished towards the equator of the eye, where perivascular sheathing was the most prominent finding (Figs. 3, 4). Many cotton-wool spots were seen in the mid and far peripheries (Figs. 4,5 ), but other than that the far periphery was not involved, and the vessels appeared normal (Fig. 5).

LABORATORY DATA

The white blood count was $4 \times 10^{9} / 1$ with less than $5 \%$ 
lymphocytes.: Haemoglobin was $9.4 \mathrm{~g} / \mathrm{dl}$, the haematocrit was $29 \cdot 8 \%$, and ESR was $54 \mathrm{~mm} / \mathrm{h}$. Blood serology revealed a CMV titre of $1: 80$ and an Epstein-Barr virus titre of 1:40. Repeated cultures of blood, cerebrospinal fluid, and bone marrow were normal. Cultures from saliva, urine, and plasma lymphocytes were all positive for CMV. Electrocardiography and chest $x$-ray revealed no abnormalities.

\section{IMMUNE STUDIES}

Skin tests with passive haemagglutinin, concanavalin$\mathrm{A}$, and Prausnitz-Küstner skin-sensitising antibodies were negative. Immunoglobulin and circulating immune complex levels were within the upper limits of normal. Phenotypic analysis of the T-lymphocyte subpopulation was performed by indirect immunofluorescence with a panel of OKT antibodies. ${ }^{10} \mathrm{Of}$ the peripheral blood lymphocytes, $38 \%$ were OKT-3 (normal 55-70\%), 9\% were OKT-4 (normal $50-60 \%$ ) and $20 \%$ were OKT-8 (normal $25-35 \%$ ). The ratio T4:T8 was $0 \cdot 5$ (normal -2 ).

\section{FOLLOW-UP}

The patient remained in hospital for several weeks, during which time he received intramuscular injections of thymic hormone factors. A slight improvement in his immunological status was noted: the OKT -4 blood lymphocytes rose to $15 \%$ and the OKT-8 to $40 \%$. However, his general condition deteriorated. He developed recurrent $P$. carinii pneumonia and a nephrotic syndrome of unknown aetiology with subsequent renal failure. On the appearance of meningism lumbar puncture and CT scan of the brain were performed, but no abnormalities were found. In December 1982 the patient died. Permission for autopsy was not granted.

\section{Discussion}

Most of the clinical data characteristic of the acquired immunodeficiency syndrome were present in this patient. Like most of the patients described in the earliest reports he was an admitted homosexual, though later reports have described the syndrome in heterosexual patients as well. Early manifestations of the disease included fever, weight loss, recurrent $P$. carinii pneumonia, and mucosal candidiasis. Bronchial brushings and cultures of urine and saliva were positive for CMV. Immunological studies showed cutaneous anergy, severe T-cell deficiency, and decreased in-vitro $\mathrm{T}$-cell proliferative responses, with a reversal of the normal ratio of helper inducer T-cell to suppressor cytotoxic cells. In contradistinction to previously reported cases, ${ }^{7}$ the levels of the IgA, IgM, IgE, and circulating immune complexes were within the upper limits of normal, and there was no clinical evidence of Kaposi's sarcoma ${ }^{45}$ The final outcome was fatal.

The aetiology of the acquired immunodeficiency syndrome is unknown. However, CMV has been incriminated by numerous authors. ${ }^{1-3671112}$ Those suffering from the disease have consistently shown positive CMV cultures from saliva, urine, semen, or bronchial brushings. ${ }^{7-9} 11$ Moreover, $\mathrm{CMV}$ has been found in the urine of $14 \%$ of healthy homosexuals as compared with negative cultures found in healthy heterosexuals. ${ }^{12}$ It is assumed that repeated sexual contacts between homosexuals may result in CMV reinfection before recovery from a condition of cellular immune dysfunction induced by a previous CMV infection, and that this may eventually lead to a chronic state of immunosuppression and immunodeficiency. This latter assumption is debatable, however, since one could instead postulate that there is a primary immunodeficiency state related to $\mathrm{T}$-cell dysfunction and that this facilitates recurrent episodes of CMV infection.

Of particular interest is the involvement of the eyes in the syndrome. It seems that at least some eye involvement is very common. ${ }^{6-9}$ In most cases it is the posterior segment that is affected, and the usual findings could be described as retinitis with differing degrees of severity. In milder cases the findings may be limited to isolated foci of cotton-wool exudates; severe cases may show a necrotising retinitis affecting all the retinal layers, mainly in the posterior pole, in which blood mixed with necrotic tissue gives the appearance of 'ketchup on cottage cheese.' In intermediate stages there may be coalescence of the exudative and necrotising patches. Perivasculitis affecting both arterioles and venules may also be found in more severely affected retinae.

The presence of $\mathrm{CMV}$ in the eye has been confirmed in only a few cases. ${ }^{89}$ Optic nerve damage has been correlated with the finding of CMV in the nerve." There is evidence to show that large exudative and necrotising retinal lesions contain viral structures. ${ }^{89}$ On the other hand the appearance of cotton-wool spots has been attributed to the presence of $P$. carinii in the inner retina. ${ }^{13}$ The extensive damage to the retina and the presence of cotton-wool spots have also been attributed to an immune complex-mediated vasculitis, since high levels of circulating immune complexes have been reported in patients with this syndrome. ${ }^{7}$ Our findings in the present case do not support this assumption.

The frequent occurrence of ocular involvement in the acquired immunodeficiency syndrome is important clinically. Such involvement seems to appear at a very early stage of the disease; thus the fundal appearance, while not pathognomonic, could be of help in diagnosis. 


\section{References}

1 Gottlich MS. Schanker HM, Fant PT, et al. Pncumocystic pncumonia. Los Angeles Morbid Mortal Weekly Rep 1981: 30: 25()-2.

2 Gottlich MS, Schroff R, Schanker HM, et al. Pneumocystis carinii pncumonia and mucosal candidiasis in previously healthy homosexual men: evidence of a new acquired cellular immunodeficiency. N Engl J Med 1981: 305: 1425-31.

3 Masur H, Michclis MA. Greenc JB, et al. An outbreak of community-acquired Pneumocystis carinii pneumonia: initial manifestation of cellular immunc dysfunction. $N$ Engl J Med 1981; 305: $1431-8$.

4 Durack DT. Opportunistic infections and Kaposi's sarcoma in homosexual men. N Engl J Med 1981 ; 305: 146.5-7.

5 Hymes KB. Cheung T. Greene JB, et al. Kaposi's sarcoma in homosexual men-a report of eight cascs. Lancet 1981; ii: $598-6(x)$.

6 Chawla HB, Ford MJ, Munro JF, et al. Ocular involvement in cytomegalovirus infection in a previously healthy male. $\mathrm{Br} \mathrm{Med} \mathrm{J}$ 1976; ii: $281-2$.

7 Holland GN. Gottlich MS, Jee RD. et al. Ocular disorders associated with a new severe acquired cellular immunodeficiency syndrome. Am J Ophthalmol 1982; 93: 393-4()2.

8 Bachman DM, Rodrigues MM, Macher AM, et al. Cultureproven cytomegalovirus retinitis in a homosexual man with the acquired immunodeficiency syndrome. Ophthalmology 1982; 89: $797-8(14$.

9 Neuwirth J. Gutman I, Odel J, et al. Cytomegalovirus retinitis in a young homosexual male with acquired immunodeficiency. Ophthalmology 1982: 89: 8(05-8.

11) Thomas J, Rogozinski L. Irigojen OH, et al. Functional analysis of human T-cell subsets defined by monoclonal antibodics. IV. Induction of suppressor cells within the OKT-4 population. $J$ Exp Med 1981: 154: 459-67.

11 Murray HW, Knox DL. Green WR, Susel RM. Cytomegalovirus retinitis in adults. A manifestation of disseminated viral infection. Am J Med 1977: 63: 574-84.

12 Drew WL, Mintz L, Miner RC, et al. Prevalence of cytomegalovirus infection in homosexual men. J Infect Dis 1981; 143: $188-92$.

13 Editorial. An analysis of retinal cottonwool spots and cytomegalovirus retinitis in the acquired immunodeficiency syndrome. Am J Ophthalmol 1982: 95: 118-9. 